Military Technical College Kobry El-Kobbah, Cairo, Egypt

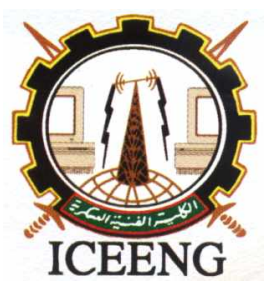

\author{
$6^{\text {th }}$ International Conference \\ on Electrical Engineering \\ ICEENG 2008
}

\title{
Microcontroller based automatic voltage regulator of synchronous generator using chopper as exciter
}

$$
\text { By }
$$

\author{
Dr. Abdelelah K. M.*
}

\section{Abstract:}

In this work a single chip microcontroller AT 89C51 is programmed in an assembly language to regulate the terminal voltage of a synchronous generator practically. The output of the microcontroller is as pulse width modulation (PWM) which drives a static exciter as power transistor chopper circuit. The PWM is generated from microcontroller utilizing the timer and programmed in such way that produces duty cycle as a function of the error between reference signal and a measured terminal voltage.

The reference voltage has a two part $8+4$ bit string and the measured also consist of two parts $8+4$ bit string, the absolute error between reference strip and measured strip is computed for each part of the signal individually.

The result shows that when a drop in terminal voltage occur the regulator reject this effect and goes back to its set value as fast as possible.

\section{Keywords:}

AVR, Microcontroller AT 89C51, Chopper, Static Exciter

* College of Engineering, Department of Electrical Engineering, University of Mosul.

** North Iraqi Petroleum Company 


\section{Introduction:}

Automatic voltage regulator (AVR) function is to process the error signal generated from the compares of reference signal with a measured and conditioned terminal voltage. After processing the error signal the output of the regulator must activate the exciter to feed a voltage or current to the winding of the generator. This voltage affects on the induced flux and so on the terminal voltage. The standard four type of regulator recommended by IEEE [1] and later researchers are deled with design [2].The AVR can be realized either by utilizing analogue devices like operational amplifiers and other electronic devices [3,4], or digitally. Several drawbacks in analogue AVR such as drift in the values of electronic and electrical elements like resistors capacitors and other elements due to surrounding environment change. Using digital AVR, these drawbacks can be avoided because it depends on software implementation of the regulator. A single chip microcontroller is nowadays is available and cheap commercially which can be programmed and implement as controller or regulator, it has a flexible property that one can change the parameters from the program. The publications on these topics, for the digital AVR implementation are by use of.Microcomputerr or microcontrollers that realize a controller algorithms or producing an actuating signal for the exciter $[4,5,6]$.

In our work a sign chip AT89C51[7] microcontroller is programmed to generate a PWM for the power transistor chopping circuit by use the timer as mode 3 .

\section{2-Hardware System Description:}

The hardware of the system has been soldered and builds on a printed card. The constructions of it consist of analogue to digital converter A/D 574, microcontroller AT89C51 and buffers 74SAL24 and optoisolater 6NB7. With static exciter ac switched power transistor (MOSFET, BUZ90A) and with suitable filter circuit. The overall circuit configuration shown in schematic diagram Fig. (1). The measured terminal voltage which is transformed from 220 to 6.5 Volt and rectified by a diode bridge and filtered which becomes as a low level voltage 8.35 Volt proportional to the terminal voltage then this becomes input to A/D 574 converter which convert it to 12 bit, word. The microcontroller reads the 12 bit signal and after processing the error signal between measured dated and referee level the program produce a pulse width modulation PWM output as a trigger to chopper circuit shown in Fig. (2-a,b) via an opt isolator6N137 to isolate between the plant and the microcontroller. 


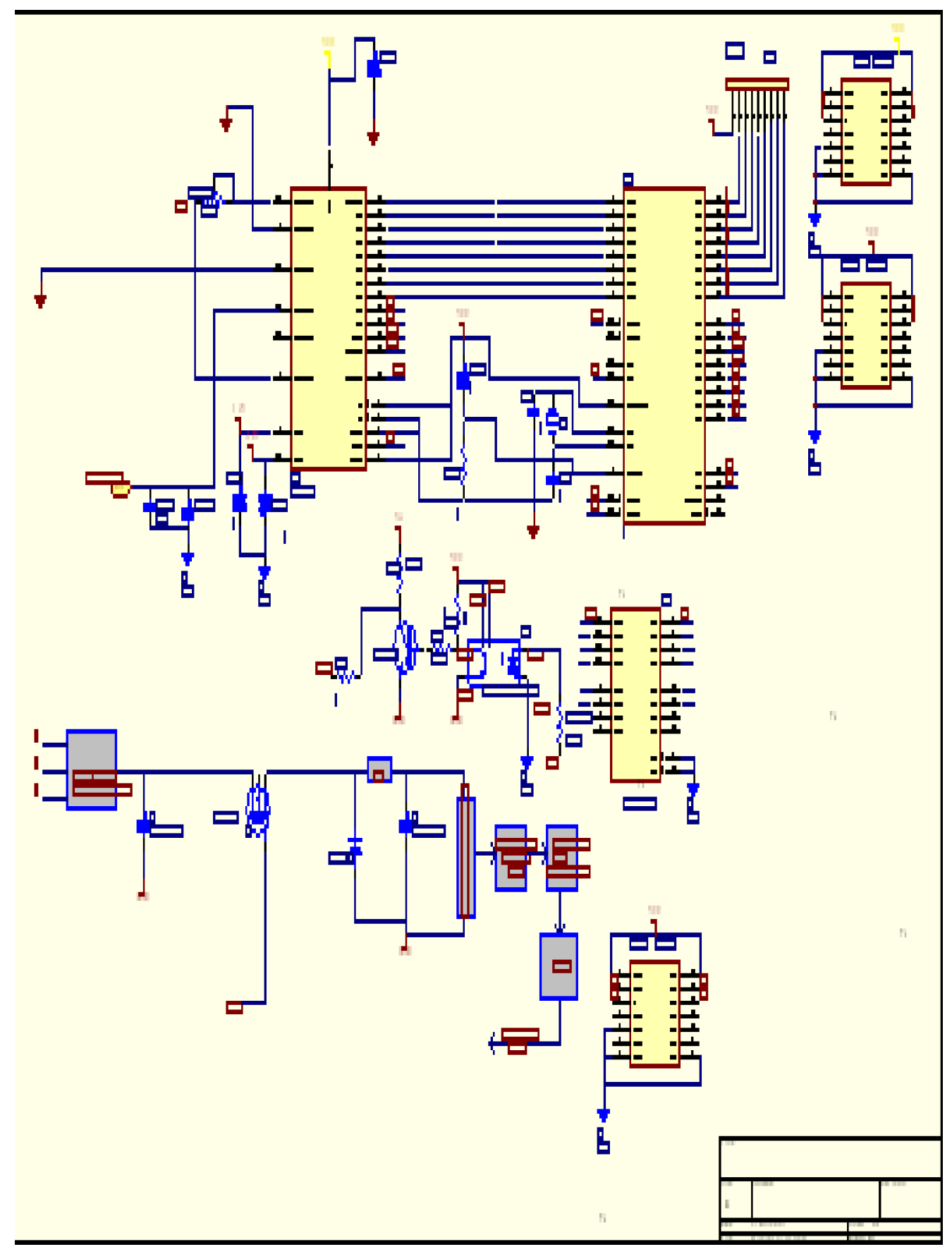

Fig. (1): The overall schematic circuit diagram 

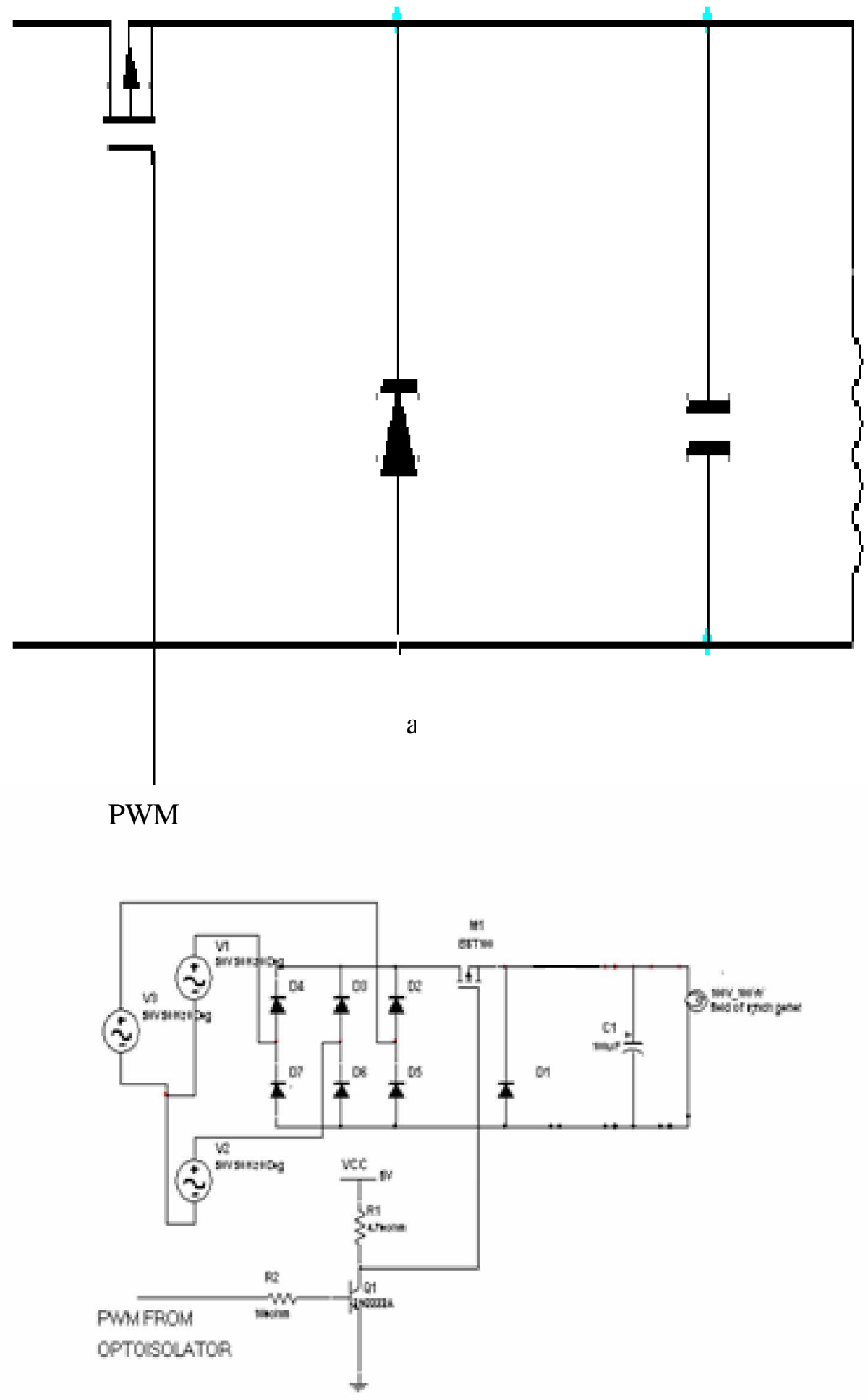

Fig. (2): a- The chopper circuit diagram

b- The chopper circuit diagram with rectifier circuit. 


\section{3-Pulse Width Modulation PWM:}

The microcontroller programmed by using the Top2005 programmer using assembly language. The program is build utilizing the timer to generate an output with different width corresponding to increase or decrease or no change in terminal voltage. The microcontroller is 8 bit input port while $A / D$ is a 12 bit so a two port used simultaneously port 1 and half of port 2 to construct a 12 bit word. The 8 bit from port 1 called $\mathrm{r} 1$ and the 4 bit called $\mathrm{r} 2$, then both $\mathrm{r} 1 \mathrm{r} 2$ construct a $\mathrm{r} 1+\mathrm{r} 2$ as a 12 bit string. The signal is compared with a reference signal which corresponds to $8.35 \mathrm{~V}$ with $5 \%$ acceptable error. The reference value becomes between two numbers $8.35 \pm 5 \%$ which is $8.3 \mathrm{~V}$ or $8.4 \mathrm{~V}$. These values converted to ob $47 \mathrm{H}$ and ob $70 \mathrm{H}$ hexadecimal. The representation for both measured string and the reference string shown in Fig. (3). When the terminal voltage has no change and then output will be a constant duty cycle or no change in the width of PWM signal. When the input data corresponding to the measured terminal voltage is changed or at occurrence of any disturbance, the duty cycle must be changed in order to reject this disturbance.

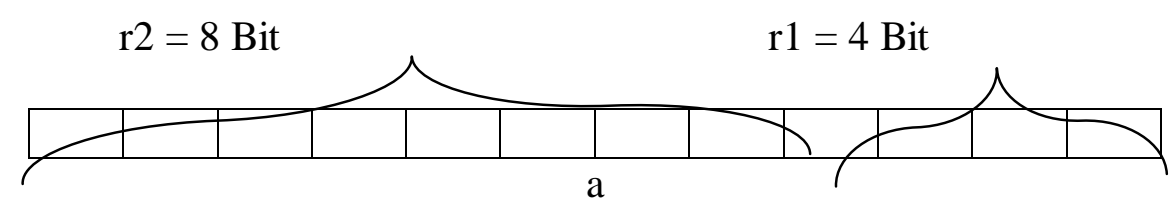

\begin{tabular}{|c|c|c|c|c|c|c|c|c|c|c|c|}
\hline 1 & 1 & 0 & 0 & 0 & 1 & 1 & 1 & 0 & 0 & 0 & 0 \\
\hline $0 \mathrm{~b} 70 \mathrm{H}$
\end{tabular}

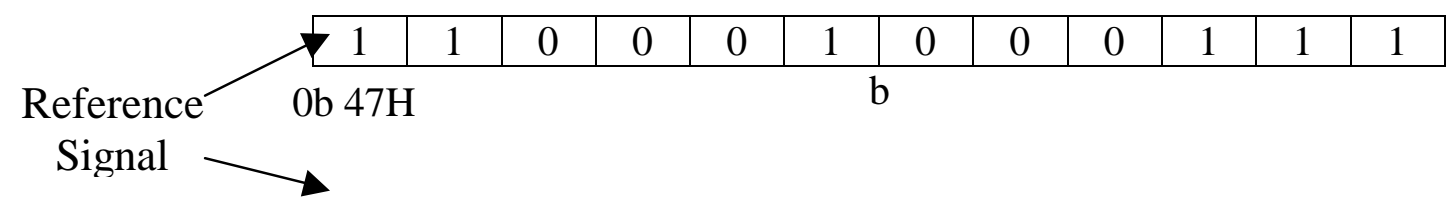

Fig. (3): a- The measured terminal voltage as two parts string chromosome b- The reference as two parts string with 5\% error. 


\section{4- Software:}

The software begins starting the program and with setting the timer to work as a mode 3. and to be interrupted externally first and to work as a timer from content of 60 $\mathrm{h}$ to FF. The second part of the program is to read the input data from the A/D converter by from ports $\mathrm{P} 1$, and half of port 2 the port $\mathrm{P} 1$ called $\mathrm{r} 2$ as 8 bit, with half port called $\mathrm{r} 1$ as 4 bit. Then the r1, r2 are combined to construct a word with 12 bit called as string or chromosome with $\mathrm{r} 1, \mathrm{r} 1$ genes. This word is compared with a reference. Signal ob $47 \mathrm{H}$ or ob $70 \mathrm{H}$. If there is no change from the reference value the timer will generate the same pulse width without change in duty cycle. The compares at beginning compares $\mathrm{r} 1$ with ob $74 \mathrm{H}$ if it is equal, then there is no carry bit and the second part $\mathrm{r} 2$ also compares with ob $70 \mathrm{H}$ if it equal, then them no change in the duty cycle remain the same. When the compares are not equal then ther will be a carry signal these will guide the program to sub program to interrupt the timer and change the beginning count time and finish time it means decrease or increase the width of the PWM output signal from port 3.6. A complete flow chart is given in Fig. (4) for the program. 


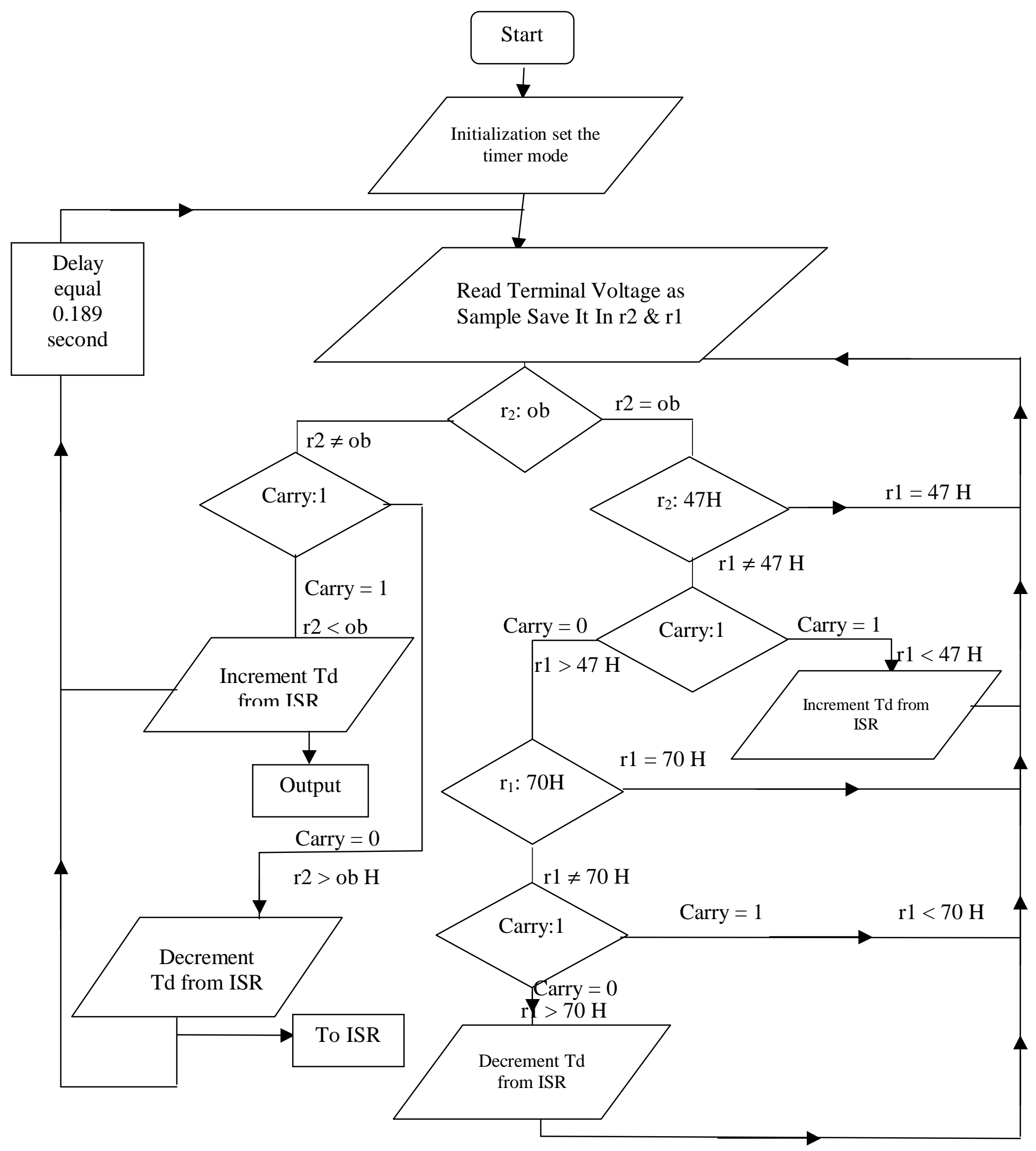

Fig. (4): The complete main program flow chart 


\section{5- Experimental Setup:}

The designed AVR card utilized to control the terminal voltage of a micro alternator with d.c. motor as a prime mover in the laboratory setup, as shown in Fig. (5). Where the terminal voltage is stepped down using voltage transformer $220 / 6.3 \mathrm{~V}$ and rectified with diode bridge and suitable filter. This signal is input to the $A / D$ converter becomes 8.35 volt with generator no load.

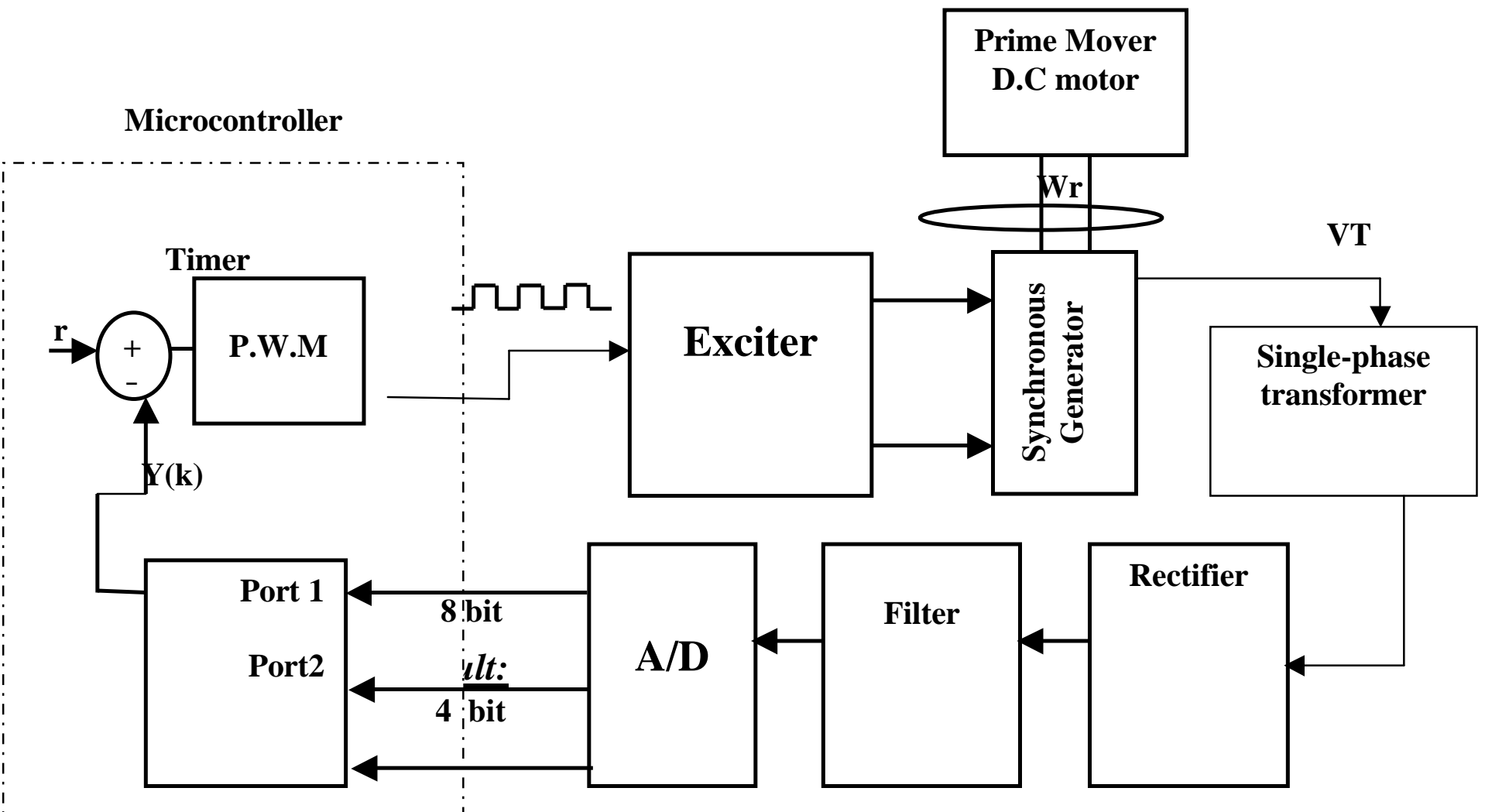

Fig. (5): Experimental Setup

When the speed of rotation of prime motor is kept constant and with no load on the generator, the pulse width signal from the output of the microcontroller pin 3.6 has a constant width, the field voltage and current is shown in fig. (6). When a resistive threephase load is applied suddenly to the generator, the terminal voltage drops for few mill second and the terminal voltage return back $t$ its original value at very short time in fig. (7) shows the terminal voltage, field voltage. 


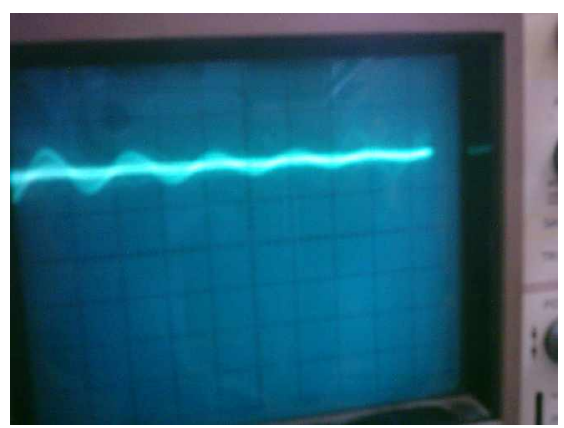

Field current waveform 0.5 volt/Div., $5 \mathrm{msec} / \mathrm{div}$

(b)

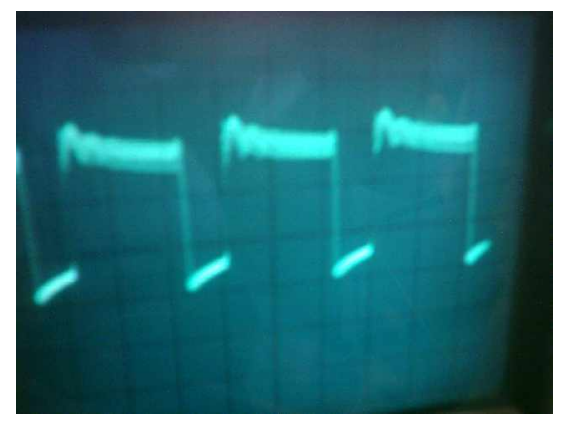

Field voltage waveform $=33$ Volt

10 volt/Div., $0.1 \mathrm{msec} / \mathrm{div}$

(a)

Fig. (6) : Field voltage and current for synchronous generator
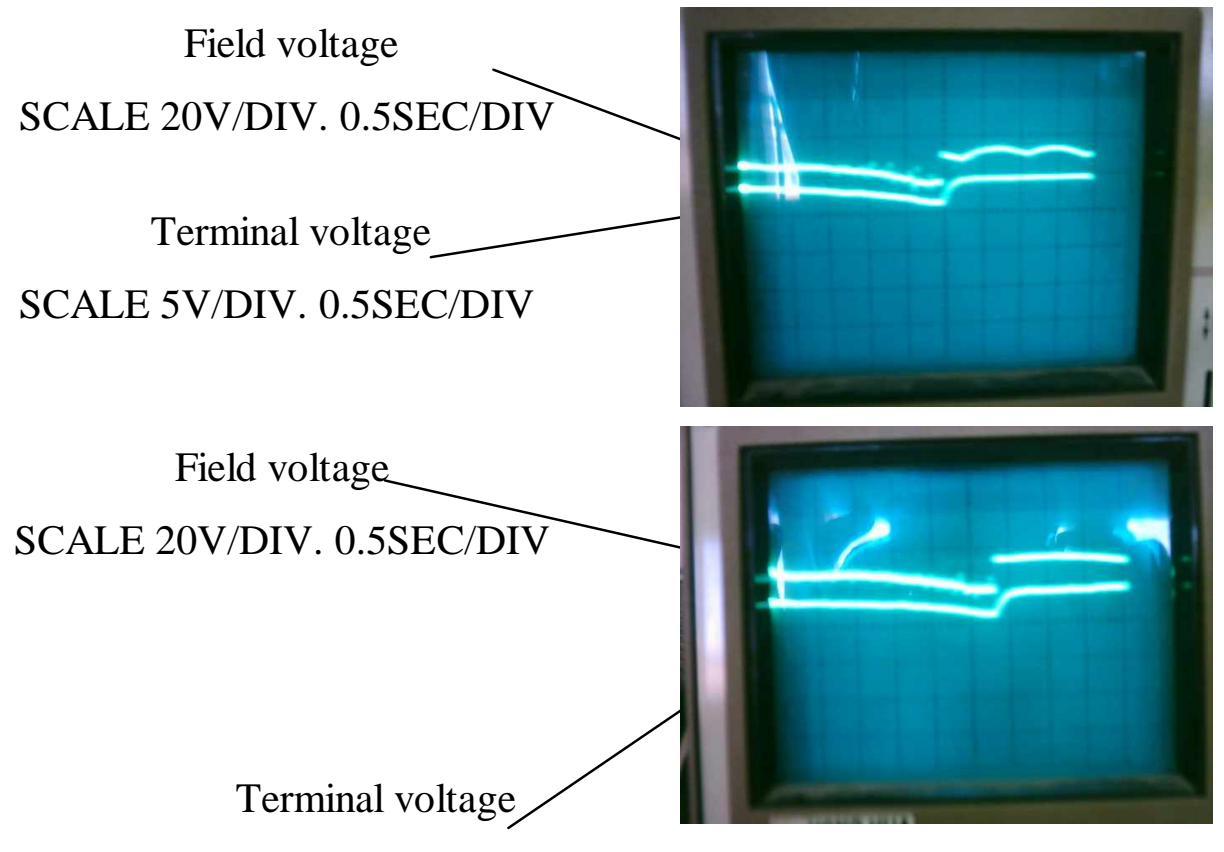

SCALE 5V/DIV. 0.5SEC/DIV

Fig. (7): Shows the terminal voltage and field voltage when a sudden resistive load applied to the generator. 


\section{6-Conclusions:}

- The timer of the microcontroller AT89C51 is programmed generate a PWM output without utilizing the saw tooth signal to produce PWM.

- The flexibility at the software program to change the content of the program to be suitable to other generators.

- The result shows that whenever a sudden load is applied to generator, the terminal voltage is automatically regulated and turns to it rated value a very fast about 0.2 sec.

- The principle of genetic algorithm is applied to the date form entered to the microcontroller when the date constructed as two part $\mathrm{r} 1 \mathrm{r} 2$ which called genes of the string or the chromosome. The absolute error signal is minimized by changing the duty cycle of PWM, with the sampled date as a population.

\section{7-References:}

1- IEEE Committee Reports "Computer Representation of Excitation System". IEEE trans. on Power Apparatus and System, Vol. PAS 87, No. 6, June. 1968.

2- C. L. Brasca \& M. A. Johnson, "On Automatic Voltage Regulator Design for Synchronous Generators", IEEE International Conference on Control and Applications, Vol. 1, No. 3, Aug. 1994.

3- R. C. Schaeter, "Applying Static Excitation Systems", IEEE Industry Application, Magazine November, December 1998.

4- Angelo J. J. rezck \& Carlos Alberto D-Coelho, "The Modulus Optimum (MO) Method Applied to Voltage Regulation Systems: Modeling, Tuning and Implementation", EFEI-ESCOLa Federal de Engenhana de Itajuba, AV. BPS, 1303 Itajuba-MG-Brazil 37500-903. E-Mail rezek@iee.efei.br.

5- Kiyons Kim. Richard C. Schaeter. "Tuning a PID Controller for a Digital Excitation Control System". IEEE Trans. On Industry Application Vol. 41, No. 2, March/April 2005.

6- A. Mirdoch and J. J. Sanchez-Gasca, M. J. D'Antonio and R. A. Lawson, "Excitation Control for High Side Voltage Regulation", Panel Session Summary for the IEEE/PES 2000 SPM, Seattle, WA.

7- Advanced Micro Devices, "Microcontroller Handbook", Intel Corporation, 1988. 